\title{
Detrimental effects of PVAT derived PGE2 and H2S in human coronary arteries with atherosclerosis
}

\author{
Gulsev Ozen ${ }^{1}$, Catherine Deschildre ${ }^{2}$, Larissa Kotelevets ${ }^{3}$, Eric Chastre ${ }^{3}$, Dan Longrois ${ }^{2,4}$, \\ Gokce Topal ${ }^{1}$, Jean-Etienne Fabre ${ }^{2}$, Jean-Baptiste Michel $^{2}$, Xavier Norel $^{2}$
}

${ }^{I}$ Istanbul University, Department of Pharmacology, Turkey, ${ }^{2}$ INSERM U1148, Paris Nord University, Sorbonne ParisCite, Paris, France, ${ }^{3}$ INSERM U1149, CNRS ERL8252, Paris Diderot University, Sorbonne Paris-Cite, Paris, France, ${ }^{4} A P-H P C H U$ X. Bichat, Department of Anesthesia and Intensive Care, Paris, France

Background: Most of the human blood vessels are surrounded by perivascular adipose tissue (PVAT). Several factors released from PVAT such as Prostaglandin E2 (PGE2) and hydrogen sulphide (H2S) or matrix metalloproteinase (MMP) could control vascular tone and vascular remodelling.

Aim of the study: we aimed to determine the effects of PGE2 and H2S derived from PVAT on the vascular wall of human coronary arteries (HCA) with or without atherosclerosis.

Methods: Atherosclerotic HCA and their PVAT were obtained after heart transplantation for myocardial ischemia while healthy HCA were dissected after non-ischemic cardiomyopathy. Using an organ bath system, cumulative dose-response curves were established in preparations with or without PVAT with selective PGE2 (EP1-4 receptors) agonists. Different pharmacological treatments were used: EP1-4 receptor antagonists, inhibitor of the enzyme responsible for H2S synthesis (CSE) or ERK inhibitor u0126. The release of H2S and the density of CSE, ERK phosphorylation were measured using polarographic and western-blot methods, respectively. EP3 receptor mRNA has been determined in HCA and their PVAT. Matrix metalloproteinase (MMP-1/-2 and their endogenous tissue inhibitors (TIMP-1/-2) were measured using ELISA kits.

Results: In the presence of PVAT, the contractile response to PGE2 was significantly reduced in healthy HCA, this reduction was abolished after incubation with theEP3 receptor antagonist, CSE or ERK inhibitors. Stimulation with EP3 receptor agonist induced CSE expression via ERK phosphorylation pathway in PVAT of healthy HCA. In addition, H2S production from PVAT were significantly decreased after EP3 receptor antagonist. The EP3 receptor mRNA and CSE expression were detected in PVAT. On the other hand, the relaxant effect released from PVAT was completely abolished in PVAT of atherosclerotic HCA because of impaired EP3-ERK-CSE pathway. In addition, increased MMP-1/-2 activities were observed.

Conclusions: Healthy PVAT released relaxant factor (H2S) via EP3 receptor expressed in PVAT and ERK phosphorylation. On the other hand, this vasorelaxant effect is abolished in atherosclerotic HCA and is accompanied by an enhanced MMP activity. This PVAT dysfunction observed in atherosclerosis could accelerate spasm and plaque rupture by increasing vascular contraction and vascular remodelling. Restoration of normal PVAT activity could be a promising therapeutic target in atherosclerotic HCA. 